\title{
Percepción de la imagen corporal en adolescentes practicantes y no practicantes de gimnasia acrobática \\ Perception of body image in adolescent participants and non-participants in acrobatic gymnastics \\ *Leopoldo Ariza-Vargas, ${ }^{* *}$ Alicia Salas-Morillas, ${ }^{* *}$ Jesús López-Bedoya, ${ }^{* *}$ Mercedes Vernetta-Santana *Universidad de Córdoba (España), **Universidad de Granada (España)
}

Resumen. Objetivo: analizar y comparar la percepción de la imagen corporal (IC) en adolescentes practicantes y no practicantes de gimnasia acrobática (GA) como actividad extraescolar. Método: estudio transversal con 38 chicas y 32 chicos (rango edad entre 14 y 17 años); el 35,72\% realizaba GA como actividad extraescolar y el 64,28\% restante no practicaban ninguna actividad extraescolar. Se registró el índice de masa corporal (IMC) y la percepción de la IC mediante el Test de siluetas de adolescentes. Resultados: al 55\% de las chicas que no practican les gustaría ser delgadas en comparación con el $22 \%$ de las que practican. La tasa de satisfacción de IC fue mayor en el grupo de práctica de GA (68\%) que en el grupo sin práctica (35\%), sin diferencias significativas. Esta satisfacción es más acentuada según el sexo, los chicos (85\%) y las chicas (61\%) que practicaron GA mostraron una mayor satisfacción frente a los chicos (36\%) y las chicas (35\%) no practicantes. El 40\% de no practicantes y el 45\% de practicantes de GA no presentan distorsión con su IC. Con respecto al sexo, las chicas GA mostraron menos distorsión (50\%) en comparación con las que no practican (35\%), siendo similares en el caso de los chicos, $42 \%$ y $44 \%$ respectivamente. Conclusiones: los adolescentes que practican GA parecen presentar mayor satisfacción y menor distorsión de su IC que aquellos que no practican esta actividad. Los chicos presentan más casos de distorsión de IC y menores tasas de insatisfacción que las chicas.

Palabras clave: gimnasia acrobática, imagen corporal, test de siluetas de adolescentes, actividad extraescolar, trastorno alimentario.

Abstract. Objective: to analyze and compare body image (BI) perception in adolescent who participante vs. do not participate in acrobatic gymnastics (AG) as an after-school activity. Method: a cross-sectional study was carried out with 38 girls and 32 boys (age range between 14 and 17 years old), out of which $35.72 \%$ performed $A G$ as an after-school activity, whilst $64.28 \%$ did not practice any extracurricular activity. Body mass index (BMI) and BI perception were recorded using the Adolescent Silhouette Test. Results: 55\% of non-practice girls would like to be thin compared to $22 \%$ of practice. BMI satisfaction rate was higher in the AG practice group (68\%) than in non-practice group (35\%), without significant differences. This satisfaction is more accentuated by gender, since boys $(85 \%)$ and girls (61\%) who practiced AG showed greater satisfaction compared to non-practice AG boys (36\%) and girls (35\%). A total of $40 \%$ of participants in the non-practice group and $45 \%$ of the AG practitioners showed no BI distortion. Regarding gender, AG girls showed less distortion (50\%) compared to those who do not practice (35\%), being this proportion similar in boys, with $42 \%$ and $44 \%$ respectively. Conclusions: adolescents who practice AG seem to present greater perception and less distortion of their BI than those who do not practice this activity. Boys present more cases of BI distortion, although dissatisfaction rates were lower than in girls.

Keywords: acrobatic gymnastics, body image, teenagers' silhouettes test, extracurricular activity, eating disorder.

\section{Introducción}

La imagen corporal (IC) representa la manera en que un individuo se percibe, imagina, siente y actúa respecto a su propio cuerpo (Trejo, Castro, Facio, Mollinedo y Valdez, 2010). Esta puede ir cambiando a lo largo de la vida, siendo más acentuada en la adolescencia por ser una etapa de grandes cambios físicos y psicológicos y de gran vulnerabilidad hacia la apariencia física por las influencias generadoras del medio ambiente en el que se desenvuelve (García y Garita, 2007). Igualmente, es una etapa donde la preocupación por agradar a los demás aumenta (Marrodán et al., 2008).

Actualmente los problemas de imagen corporal, que conllevan una distorsión e insatisfacción con la IC, surgen generalmente por la presión social de los modelos estándares de belleza que establece la sociedad actual (Vaquero-Cristóbal, Alacid, Muyor y Loìpez-Miñarro, 2013).

La mayoría de los estudios sobre la identificación de posibles trastornos de la IC, se han centrado en adolescentes, por ser una de las etapas más vulnerables a los ideales de delgadez para el sexo femenino y un cuerpo musculoso y atlético para el sexo masculino que promueve la sociedad

Fecha recepción: 09-03-20. Fecha de aceptación: 06-05-20 Leopoldo Ariza Vargas

eolarval@uco.es actual (De Gracia, Marcó y Trujano, 2007). Una preocupación excesiva por alcanzar un físico ideal en estas edades, puede desencadenar trastornos de conducta alimentaria (TCA), insatisfacción y distorsión de la imagen, y baja autoestima, obsesión por la delgadez, provocando graves problemas sobre la salud (Vaquero-Cristóbal et al., 2013; Tylka y Wood-Barcalow, 2015; Arrayás, Tornero y Díaz, 2018).

Por otro lado, varios estudios evidencian que la práctica de la actividad física y/o deportiva en los adolescentes se relacionan con una buena IC y una mejor percepción de siì mismos (Contreras, Fernández, Gracía, Palou y Ponseti, 2010). No obstante, no existe un consenso al respecto, pues algunos autores como Gonález-Montero et al. (2010), indican que depende del tipo de actividad física realizada, mientras que otros, sostienen que, en deportes con predominio estético, suele prevaler una carga negativa sobre la auto-valoración corporal, siendo los deportes gimnásticos en general uno de ellos (Vaquero-Cristobal et al., 2013). En este último caso, las discrepancias son mayores, ya que son varios los autores que indican que los practicantes de deportes con componente artístico entre los que se encuentran los deportes gimnásticos son los que presentan una mayor estima corporal (Miñano, García y Galiano, 2006; Vernetta, Fernández, LópezBedoya, Gómez-Landero y Oña, 2011; Del Pozo, Piedra y Alfonso, 2017; Peláez y Vernetta, 2018; Vernetta, Montosa y Peláez, 2018). 
De acuerdo a lo expuesto, nuestro interés se ha centrado en comparar la percepción de la IC en adolescentes practicantes y no practicantes de Gimnasia Acrobática (GA) como actividad extraescolar.

La GA es actualmente una disciplina gimnástica eminentemente cooperativa donde la unión de elementos técnicos grupales e individuales unidos y sincronizados a un soporte musical mediante una coreografía, constituye la esencia de esta disciplina deportiva, de ahí, que el componente artístico tenga un lugar clave en esta modalidad (Vernetta, LópezBedoya y Gutiérrez, 2008). Es un deporte estético donde la IC, el peso, un índice de masa corporal (IMC) y un porcentaje de grasa bajo, junto a otros múltiples factores, son algunos de los determinantes en el éxito deportivo (Taboada-Iglesias, Vernetta y Gutiérrez-Sánchez, 2017).

Son pocos los estudios realizados en este deporte sobre la percepción de la IC, no existiendo ninguno que haya aplicado el Test de Siluetas en Adolescentes (TSA) de Maganto y Cruz (2008), el cual se trata de un test más evolucionado que los clásicos test de figuras de Stunkard y Stellar(1990) y Collins (1991), utilizados para obtener la percepción, distorsión e insatisfacción de la IC, ya que no solo centra la atención en analizar la figura percibida y deseada que le gustaría tener, sino que añade otra serie de preguntas relacionadas con la figura que elegirían como pareja y la que elegiría el sexo contrario, así como, la figura que les gustaría a sus progenitores que tuvieran. Con lo cual, tiene en cuenta en parte, la presión que puede ejercer su familia y compañero del sexo contrario, siendo uno de los factores también influyentes en la preocupación corporal de estos adolescentes (Cash y Pruzinsky, 1990).

De ahí, que el objetivo de este estudio haya sido conocer la percepción de la IC en estudiantes adolescentes entre 14 y 17 años de ambos sexos en función de si practican o no GA. El hecho de incluir la GA como actividad extraescolar, es porque pensamos que puede ser un deporte beneficioso para la percepción de la IC, además de ser una modalidad inclusiva para ambos géneros. Como objetivos específicos se han planteado: a) comparar el grado de satisfacción con respecto a la IC que poseen los adolescentes practicantes y no practicantes de GA de ambos sexos y b) analizar y comparar la distorsión de la IC en gimnastas de acrobática en relación al sexo con los adolescentes no practicantes de este deporte.

\section{Material y método}

\section{Diseño y participantes}

Estudio observacional descriptivo de corte transversal y comparativo, con la participación voluntaria de 70 adolescentes (38 chicas y 32 chicos), cuya selección fue intencional. Del total de la muestra, el 35,71\% realizaba gimnasia acrobática como actividad extraescolar dos veces por semana durante dos horas en diferentes clubs de gimnasia. El $64,28 \%$ restante, todos adolescentes de un instituto público de Granada no practicaban ninguna actividad extraescolar. La selección de la muestra atendió a criterios de accesibilidad con edades comprendidas entre los 14 y 17 años, tras obtener la firma del consentimiento informado de sus padres/madres o tutores legales.

\section{Instrumentos}

Se pasó una hoja de auto-registro con preguntas relativas a características físicas y sociodemográficas, incluyendo la edad, nivel y curso académico, y preguntas sobre si practicaban alguna actividad deportiva y en caso afirmativo, años de práctica, club, nivel competitivo y horas semanales de entreno.

En segundo lugar, para medir la variable percepción de la IC se aplicó el TSA de Maganto y Cruz (2008), desarrollado para evaluar los aspectos más relevantes de la IC específicamente en adolescentes de 14 a 18 años de edad.

El test está compuesto por ocho figuras masculinas y ocho figuras femeninas que muestran un incremento progresivo del tamaño corporal en anchura de cada una de ellas. Consta de 8 preguntas: la primera se responde en base a lo que observa en las siluetas masculinas, otorgándole un adjetivo a cada una (delgada, normal o gorda), la segunda sigue el proceso anterior, pero en este caso se divisan siluetas femeninas a las que igualmente se les adjudican cualidades como delgada, normal o gorda. La pregunta tercera evalúa la propia percepción de IC, la cuarta identifica la silueta o el IMC deseado, la quinta la silueta que elegiría como pareja, la sexta la silueta que el sexo contrario elegiría como pareja, la pregunta séptima, las atribuciones de las preferencias de la madre, y la octava, las atribuciones a la preferencia del padre.

Con las preguntas 3 y 4 se halla el índice de distorsión e insatisfacción de la imagen corporal. A cada figura se le otorga un intervalo de valores de adecuación o no del IMC esperable a su edad clasificadas en categorías de normales, delgadas y gruesas (Maganto y Cruz, 2008).

Un valor 0 indica que no hay distorsión de la imagen corporal, mientras que los valores negativos supone que los estudiantes se ven más delgados de lo que realmente son y los valores positivos que se observaba con un mayor IMC del que realmente tiene. Se considera con un alto riesgo de sufrir TCA aquellos estudiantes o deportistas con puntuaciones mayores de 3 puntos, de acuerdo con el baremo establecido por los autores (Maganto y Cruz, 2008).

Finalmente, para las variables antropométricas, la estatura fue medida utilizando un tallímetro (SECA220 con precisión de $1 \mathrm{~mm}$ ) y la masa corporal, se determinó con una balanza SECA(713, Hamburg, Alemania, con una precisión de $0,1 \mathrm{~kg})$. Se calculó el IMC como la masa corporal dividida por la altura elevada al cuadrado $\left(\mathrm{Kg} / \mathrm{m}^{2}\right)$.

\section{Procedimiento}

Previamente a la toma de datos, para solicitar la colaboración de los participantes, se elaboró una carta informativa del objetivo del estudio, la cual se facilitó al director del centro educativo y a los entrenadores de los clubs deportivos. En este escrito se solicitaba el consentimiento informado para participar en este estudio. Posteriormente, se aplicó el cuestionario de auto registro y seguidamente el TSA. La recogida de datos se realizó en el propio centro educativo en el curso académico 2018/19 durante la primera semana de abril en el horario lectivo de las clases de educación física de 10:15 a 14:45 horas y a los gimnastas en los diferentes clubs de gimnasia durante la segunda semana de abril, en su horario de actividad de 17:30 a 19:30 horas.

Los test se realizaron en grupos de 10 a 15 integrantes en 
muestran distintas en chicas y en la muestra total, según practiquen o no actividad física: $\chi^{2}(3, \mathrm{~N}=38)=10,132, p=, 017$ y $\chi^{2}(4, N=70)=9,893, p=, 042$ respectivamente, $\mathrm{y}$ en los participantes no practicantes de actividad física y la muestra total, según sean chicos o chicas: $\chi^{2}(3, \mathrm{~N}=45)=10,038, p=$ , 018 y $\chi^{2}(4, \mathrm{~N}=70)=10,159, p=, 038$ en el mismo orden (véase Tabla 2).

\section{Análisis de los Índices de Distorsión y de Insatisfac- ción}

En la Tabla 3 se muestra la distribución del índice de distorsión, según los pares constituidos por las categorías de las variables Práctica y Sexo. El estadístico U de Mann Whitney no mostroì diferencias estadísticamente significativas entre las distribuciones del índice de distorsión entre ninguno de los pares resultantes (véanse Tablas 3 y 4).

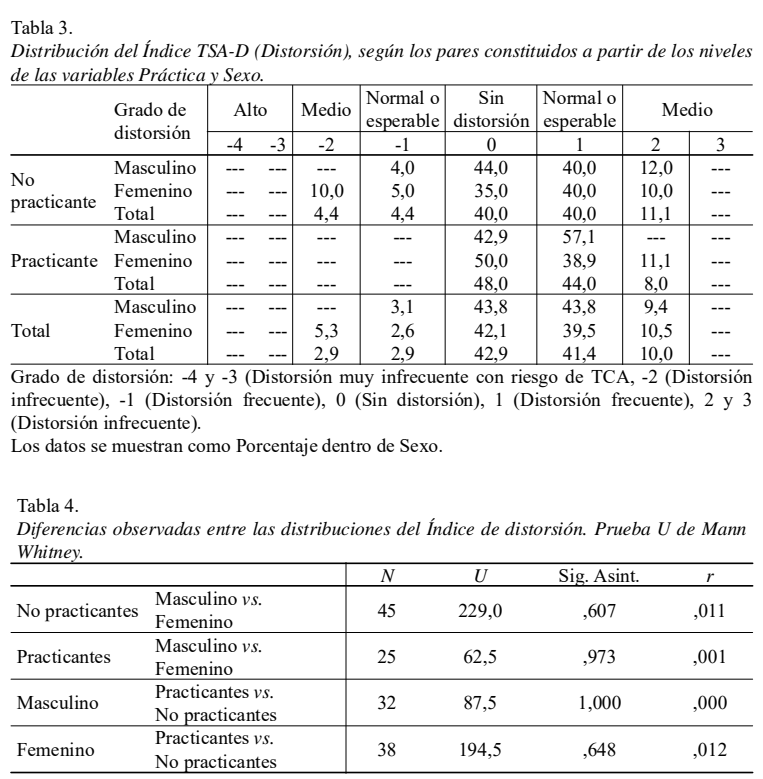

La distribución del índice de insatisfacción, según los pares constituidos por las categorías de las variables Práctica y Sexo, se recoge en la Tabla 5. El estadístico U de Mann Whitney no mostró diferencias estadísticamente significativas entre las distribuciones del Índice de insatisfacción entre ninguno de los pares resultantes (véase Tabla 6).

Tabla 5 .

Distribución del Índice TSA-I (Insatisfacción) según los pares constituidos a partir de los niveles de las variables Práctica y Sexo.

\begin{tabular}{|c|c|c|c|c|c|c|c|c|c|c|}
\hline & & \multicolumn{9}{|c|}{ Grado de insatisfacción } \\
\hline & & \multicolumn{2}{|c|}{ Grado alto } & \multicolumn{2}{|c|}{$\begin{array}{l}\text { Normal o } \\
\text { esperable }\end{array}$} & \multirow{2}{*}{\begin{tabular}{|c} 
Satisfacción \\
0
\end{tabular}} & \multirow{2}{*}{$\begin{array}{c}\begin{array}{c}\text { Normal o } \\
\text { esperable }\end{array} \\
1\end{array}$} & \multicolumn{2}{|c|}{ Grado medio } & \multirow{2}{*}{ Total } \\
\hline & & -4 & -3 & -2 & -1 & & & 2 & 3 & \\
\hline \multirow{3}{*}{$\begin{array}{l}\text { No } \\
\text { practicante }\end{array}$} & $\mathrm{M}$ & --- & --- & $2(8,0)$ & $5(20,0)$ & $9(36,0)$ & $7(28,0)$ & $2(8,0)$ & --- & $25(100)$ \\
\hline & $\mathrm{F}$ & -- & -- & ---1 & $11(55,0)$ & $7(35$ & $2(10)$ & -- & --- & \\
\hline & Total & -.- & -.- & $2(4,4) 1$ & $16(35,6)$ & & $9(20$, & $2(4,4)$ & -- & $45(100)$ \\
\hline \multirow{3}{*}{ Practicante } & $\mathrm{M}$ & --- & --- & \begin{tabular}{|c|}
--- \\
\end{tabular} & -- & $6(85,7)$ & $1(14,3)$ & --- & --- & $7(100)$ \\
\hline & & --- & & --- & $4(22,2)$ & & & --- & --. & \\
\hline & Total & --- & --- & --- & $4(16,0)$ & $17(68,0)$ & $4(16,0)$ & -- & --- & $25(100)$ \\
\hline \multirow{3}{*}{ Total } & M & --- & --- & $2(6,3)$ & $5(15,6)$ & & & $2(6,3)$ & --- & \\
\hline & $\mathrm{F}$ & --- & --- & $-\ldots \mid 1$ & $15(39,5)$ & $18(4$ & $5(13$ & -- & --- & $38(10$ \\
\hline & Total & --- & -- & $\left.2(2,9)\right|_{2}$ & $20(28,6)$ & $33(47,1)$ & $13(18,6)$ & $2(2,9)$ & -.- & $70(100)$ \\
\hline
\end{tabular}

Grado de insatisfacción: $-4 \mathrm{y}-3$ (Insatisfacción muy infrecuente con riesgo de TCA), $-2 \mathrm{y}$ 1(Insatisfacción frecuente), 0 (Satisfacción), 1 (Normal o esperable), 2 y 3 (Grado medio). Los datos se muestran como Frecuencia (Porcentaje dentro de Sexo)

$\mathrm{M}=$ =masculino, $\mathrm{F}=$ femenino

Tabla 6 .

Diferencias observadas entre las distribuciones del Índice de Insatisfacción. Prueba U de Mann Whitney.

\begin{tabular}{|c|c|c|c|c|c|c|}
\hline & & & $N$ & $U$ & Sig. Asint. & $r$ \\
\hline No practicantes & $\begin{array}{l}\text { Masculino } \\
\text { Femenino }\end{array}$ & vs. & 45 & 169,0 & ,051 & ,043 \\
\hline Practicantes & $\begin{array}{l}\text { Masculino } \\
\text { Femenino }\end{array}$ & vs. & 25 & 52,5 & ,440 & ,030 \\
\hline Masculino & $\begin{array}{l}\text { Practicantes } v s . \\
\text { No practicantes }\end{array}$ & & 32 & 88,5 & ,961 & ,002 \\
\hline Femenino & $\begin{array}{l}\text { Practicantes } v s . \\
\text { No practicantes }\end{array}$ & & 38 & 238,5 & ,061 & ,049 \\
\hline
\end{tabular}

\section{Discusión}

El objetivo principal del estudio fue conocer la percepción de la IC en estudiantes adolescentes entre 14 y 17 años de ambos sexos en función de si practican o no GA. Del conjunto de datos analizados los hallazgos de mayor relevancia muestran que (1) La mayoría eligen las figuras 4 y la 5 como representante de su peso actual tanto en chicos como en chicas sin diferencias significativas entre sexo y entre practicantes de GA y no practicantes; (2) Las figuras deseadas igualmente están entre la 4 y la 5 , siendo la figura 4 en mayor medida; (3) El grado de satisfacción es mayor en las practicantes de GA aunque sin diferencias estadísticamente significativas. Esta satisfacción es más acusada cuando se diferencia por sexos siendo los chicos de GA los que presentan mayor satisfacción, no presentando ningún nivel de insatisfacción, mientras que el nivel de insatisfacción de las chicas fue esperable, ubicándose solo una silueta por debajo del índice 0 de insatisfacción con el $22 \%$. En los no practicantes, este índice de insatisfacción fue mayor tanto en chicos como en chicas; (4) Ninguno de los participantes, se encuentran en riesgo de sufrir un TCA por índice de insatisfacción de hasta dos siluetas por debajo de la que se percibe; y (5) Las chicas de GA presentaron menos distorsión en relación a los chicos, siendo a la inversa en los no practicantes, no obstante, no se apreciaron diferencias significativas entre ambos grupos ni en relación al género.

Antes de analizar y discutir de forma pormenorizada cada una de las respuestas de los ocho ítems del TSA, indicar, que los estudios realizados con el mismo son escasos, por lo que hemos tenido limitación al realizar comparaciones de nuestros adolescentes del estudio con otros trabajos similares.

$\mathrm{Al}$ analizar la primera pregunta del test referida a la imagen masculina, tanto en practicantes como no practicantes de GA en función del sexo destacar que existe unanimidad en que la figura 1 , es considerada 'delgada' y la número 8 'gorda' por los practicantes y por los no practicantes.

Para el resto de figuras, e indistintamente de la condición de práctica, la tendencia observada es similar (véase tabla 1). Destacan las figuras 2 y 3 como representativas de una imagen corporal delgada, las figuras 6 y 7 propias de una imagen corporal gorda y las figuras centrales 4 y 5 definitorias de una imagen corporal normal. En el caso de las imágenes corporales delgada y gorda, los porcentajes son tanto más elevados cuanto más extrema es la figura. Es decir, a tenor de la frecuencia de elección observada, y exceptuando las representaciones 1 y 8 , las figuras 2 y 7 (con valores porcentuales próximos o superiores a 90) reflejan mejor una imagen corporal delgada y gorda que las figuras 3 y 6 respectivamente.

Con respecto a la pregunta 2 , relacionada con las figuras femeninas destacar que no se aprecian diferencias significativas con respecto al sexo ni el nivel la práctica de GA. Existe unanimidad en la consideración de las figuras 1 y 2 como delgadas y de la figura 8 como gorda. La figura 7 también es mayoritariamente considerada como propia de una mujer de silueta gorda indistintamente del sexo y nivel de práctica con un rango de valores porcentuales observados entre 90 y 100 (véase tabla1). Respecto al perfil normal femenino, una vez más, son las figuras centrales 4 y 5 las que recogen mayor frecuencia de observaciones, sin distinción por sexo o nivel 
de práctica, con una horquilla de porcentajes que varían entre $85,7 \%$ (figura 5) y $100 \%$ (figura 4 ), en ambos casos registrados en chicos practicantes.

En cuanto a las respuestas dadas a los ítems 3 - 8 del Test de Siluetas (véase tabla 2), debemos destacar que únicamente se han encontrado diferencias significativas entre las distribuciones de las respuestas dadas a la pregunta 7 .

En relación a la pregunta 3, referente a la propia percepción de su imagen corporal, un porcentaje mayoritario de la muestra $(44,3 \%)$ tiende a elegir la figura 4 seguida de la figura $5(31,4 \%)$ como representativas de su peso actual, figuras correspondientes a un IMC dentro de la normalidad, sin llegar a poder concluir la significatividad de las diferencias observadas. Nuestros resultados no corroboran lo indicado por Marrodán et al. (2008), de que la percepción correcta es mayor en mujeres, ni tampoco los estudios que muestran que las mujeres presentan una percepción errónea de su IC (Maganto y Cruz, 2008). Sin embargo, son coincidentes con el estudio de Vernetta et al. (2018), que muestran que las gimnastas de acrobática, estaban muy satisfechas con su aspecto, su físico, así como su peso corporal, presentando todas una buena estima corporal.

En lo concerniente a la figura deseada (pregunta 4), la mayoría de los participantes tienen propensión a elegir la figura $4(67,1 \%)$, seguida de la figura 5 , si bien las chicas $(78,9 \%)$ tienden en mayor medida que los chicos $(53,1 \%)$ a identificarse con la figura 4. Hay estudios que afirman que las mujeres, aunque se perciban con una IC saludable, desean tener una figura más delgada, es decir con un IMC menor (González-Montero et al., 2010). Esto no coincide con la muestra de nuestro estudio ya que la mayoría desea tener una figura 4 o 5 que corresponde a un IMC normal (Maganto y Cruz, 2008) según los criterios de la OMS.

Las preguntas 5 y 6 indican la figura que elegirían como pareja y la que elegiría el sexo contrario como pareja, respectivamente. En ambas preguntas, se señalan las figura 4 y 5 como las elegidas. En respuesta a la pregunta 5, la figura 4 parece ajustarse mejor al ideal de pareja de los practicantes de GA y la figura 5 a los no practicantes. En la pregunta 6, la elección de la figura 4 fue más acentuada en chicas respecto de los chicos no practicantes, invirtiéndose dicha tendencia en el caso de practicantes de GA.

En la pregunta 7 ¿Qué figura le gustaría a tu madre que tuvieras?, se aprecian diferencias significativas entre sexos y practicantes de GA y no practicantes, siendo la figura más elegida la número 4 para las chicas y chicos en los practicantes de GA y la número 5 para los chicos y chicas en los no practicantes.

Por último, en la pregunta 8 ¿Qué figura le gustaría a tu padre que tuvieras?, una vez más son las figuras 5 y 4 las más escogidas por los participantes. Según el nivel de práctica, los no practicantes se decantaron preferentemente por la figura 5 mientras que los practicantes de GA optaron por la figura 4. Las respuestas de las dos preguntas últimas, no corroboran la posible relación entre la influencia de los padres en los hijos por la búsqueda de esos ideales de belleza de delgadez, ya que tanto padres como madres, marcan figuras correspondientes a IMC normal (Quiles, Quiles, Pamies, Botella y Treasure, 2013).

En relación con el índice de distorsión que relaciona la pregunta 3 con el IMC real, señalar que el $40 \%$ de los no practicantes y el $45 \%$ de los practicantes de GA no presentan distorsión con su imagen corporal. Las que menos distorsión presentan son las chicas de GA con un $50 \%$ frente a los $35 \%$ de las no practicantes. Los chicos tienen porcentajes similares de $42 \%$ y $44 \%$ respectivamente.

Estos resultados están avalados por estudios precedentes que afirman que las mujeres tienen una percepción adecuada, es decir, son las que menos distorsión presentan (Marrodán et al., 2008; Contreras et al., 2010; Vernetta et al., 2018). Igualmente, nuestras gimnastas practicantes, reiteran los resultados del estudio de Vernetta et al. (2018), quienes destacan que las de acrobática y rítmica, presentan una buena percepción de la IC, siendo menor la distorsión en las primeras.

Finalmente, el índice de satisfacción que relaciona la pregunta 4 (figura deseada) con el IMC real; es mayor en las practicantes de GA con el $68 \%$ frente a los $35 \%$ en los no practicantes, pero sin diferencias significativas. Esta satisfacción es más acusada cuando se diferencia por sexos siendo los chicos de GA los que presentan una mayor satisfacción con un porcentaje del $85 \%$ frente al $36 \%$ de los no practicantes y en las chicas de GA con un $61 \%$ frente a los $35 \%$ de las no practicantes. Aunque no existen diferencias significativas entre practicantes y no practicantes, los datos en porcentajes de satisfacción son mayores en los practicantes de GA, con lo cual se intuye esa relación positiva entre AF e IC, respaldadas en trabajos precedentes (Gómez y Núñez, 2007; Miñano et al., 2006; Contreras et al., 2010; González-Montero et al., 2010; Urrutia, Azpillaga, Luis de Cos y Muñoz, 2010).

La insatisfacción, es mayor en chicas que en chicos y en los practicantes de GA, donde un $22,2 \%$ desearían estar más delgadas frente a ninguno de los chicos. En los no practicantes, un poco más de la mitad de las chicas (55\%) desearían estar más delgadas y un $20 \%$ en los chicos. Estos datos como indican Maganto y Cruz (2008), vienen a confirmar que la cultura de la delgadez imperante en nuestra sociedad afecta preferentemente a las mujeres. Igualmente, son corroborados por Arrayás et al. (2018), quienes afirman que la mayoría de chicos y chicas adolescentes desean perder peso, siendo las chicas las que presentan mayor obsesión por la delgadez.

Por otro lado, Valles, Hernández, Baños, MoncadaJiménez y Rentería (2020), en un estudio que comparan gimnastas con un grupo control observaron mayor insatisfacción en los gimnastas que en el grupo control, al desear una silueta más delgada las chicas, mientras que los chicos una silueta más gruesa equivalente a más musculosa. Nuestros hallazgos apoyan estos resultados en cuanto a las chicas gimnastas, ya que un porcentaje muy elevado, deseaban tener la figura 4, no siendo así para los chicos quienes ninguno, querían tener una silueta más gruesa.

No obstante, resaltar que ninguno de los participantes de la muestra total presentó un índice de insatisfacción elevado, sino que simplemente, mostraron una leve tendencia a querer estar más delgados, por lo cual ninguno alertó de posibles riesgos de padecer TCA (Maganto y Cruz, 2008). Estos resultados igualmente se alejan de los reportados por Valles et al. (2020), donde los gimnastas presentaron un mayor riesgo de anorexia en comparación con el grupo control. 


\section{Conclusiones}

A nivel general podemos concluir que de las ocho figuras que componen el TSA existe unanimidad, en el total de la muestra, al considerar los perfiles corporales representados por las figuras 1 como delgada y la 8 como gorda, indistintamente de que se traten de figuras masculinas o femeninas (ítems 1 y 2). Los adolescentes tienden a verse representados preferentemente a través de los perfiles de las figuras $4 \mathrm{y}$ 5 , no existiendo diferencias significativas entre chicas y chicos. Los perfiles de estas dos últimas figuras son las más deseadas, prevaleciendo la número 4 sobre la número 5.

En relación a las figuras que elegirían como pareja y que el sexo contrario elegiría como pareja se ubican en ambos casos en las figuras 4 y 5 .

En cuanto a las preferencias de los progenitores, tanto madres como padres, coinciden una vez más en las figuras 4 y 5 , consideradas ambas con un IMC dentro de la normalidad. No obstante, en las madres, se aprecian diferencias significativas en cuanto al género, eligiendo en un porcentaje mayor la figura 4 como ideal en los practicantes de GA en ambos sexos y la figura 5 para los chicos no practicantes.

Según el índice de distorsión (relación del IMC con el ítem 3) e insatisfacción (relación del IMC con el ítem 4) en términos generales se puede concluir que se observa una tendencia no significativa según la cual las chicas practicantes presentan menor distorsión frente a las no practicantes. Se identificaron más casos de distorsión de la IC en chicos que en chicas, tanto en practicantes como no practicantes. La insatisfacción, es mayor en chicas que en chicos y en los no practicantes de GA. Más de la mitad de las chicas no practicantes (55\%) desearían estar delgadas frente al (22\%) de las chicas de GA.

Se puede indicar que aunque existe un índice de distorsión, este es bajo; y el índice de insatisfacción se encuentra dentro de lo esperable, siendo además nulo el riesgo de sufrir un TCA por los adolescentes encuestados.

Como limitaciones del presente estudio, destaca el bajo número de participantes sobre todo en el grupo de practicantes masculinos de GA, debido al bajo número de practicantes del sexo masculino en esta disciplina. Por ello, en futuras investigaciones sería interesante aumentar la muestra, especialmente en el sexo masculino de los practicantes de GA que ha sido menos numerosa, incluso hacer una diferenciación por tramos de edad (12-13; 14-15; 16-17). Igualmente, se propone introducir una comparativa de muestras de practicantes de GA de diferente nivel competitivo.

\section{Aplicaciones prácticas}

La principal aportación de este estudio ha sido observar las diferencias existentes en la percepción de la IC entre practicantes y no practicantes de GA. El estudio no presenta diferencias significativas, pero observamos una tendencia según la cual las chicas practicantes tienen un grado menor de distorsión frente a las no practicantes. De la misma manera, y en relación con el grado de satisfacción observamos que tanto chicos como chicas practicantes se encuentran más satisfechos con su IC con respecto a las no practicantes.
Estos datos sugieren, la necesidad de desarrollar intervenciones educativas con programas específicos desde la asignatura de Educación Física dirigidas a favorecer la percepción de una IC más positiva en estos adolescentes, con el fin de acrecentar la satisfacción con su IC. Se tiene constancias que estos programas con planteamientos cooperativos pueden ser eficaces en la mejora de la satisfacción corporal, autoestima, la autoevaluación del aspecto físico y además pueden modificar la susceptibilidad de los adolescentes a la presión de pares y las normas culturales relativas a la imagen corporal (Odea y Abraham, 2000; Jassen y Leblanch, 2010).

Existen evidencias que la práctica de deportes gimnásticos relacionados con la GA y rítmica ayuda a la mejora de la percepción corporal (Miñano et al., 2006; Reguera y GutiérrezSaìnchez, 2015; Vernetta et al., 2018; Peláez y Vernetta, 2018).

De ahí, que el profesorado de Educación Física puede desarrollar un papel importante en el desarrollo de la percepción de la IC de su alumnado, planteando intervenciones en su propia clase, mediante la organización de Unidades Didácticas de deportes gimnásticos con adaptaciones educativas como puede ser el Acrosport denominación de la GA en el ámbito escolar (Vernetta, López Bedoya y Panadero, 2007). Este contenido, por sus características cooperativas e integradoras, permite que los estudiantes aprendan a valorar las posibilidades de su cuerpo sin existir discriminación alguna, ya que todos son imprescindibles para conseguir éxito en las tareas propuestas, convirtiéndolo en un instrumento de gran valía dentro de las clases de Educación Física. Igualmente, se puede promover la adherencia a este contenido, mediante la organización de visitas o talleres en clubes deportivos de naturaleza gimnástica, con el fin de motivar al alumnado en su práctica como actividad extraescolar por sus múltiples beneficios psicosociales: autoestima, esfuerzo, responsabilidad, cooperación, expresión emocional, así como el desarrollo de las funciones cognitivas, motoras y afectivas (Dowdell, 2013; Ávalos, Martínez y Merma, 2015; Reguera, Gutiérrez-Sánchez y Portela, 2015).

\section{Agradecimientos}

A los entrenadores y gimnastas de los clubes participantes, así como a los adolescentes y director del centro educativo.

\section{Referencias}

Arrayás, M.J., Tornero, I., y Díaz, M.S. (2018) Percepción de la imagen corporal en adolescentes de Huelva atendiendo al género y a la edad. Retos: nuevas tendencias en educación física, deporte y recreación, 34(1), 40-43.

Ávalos, M. A., Martínez, M. A. y Merma, G. (2015). La pertinencia educativa de las habilidades gimnásticas: apreciaciones del profesorado Apunts. Educación Física y Deportes, 121, 28-35.

Cash, T.F., y Pruzinsky, T. (1990). Body Images: Development, Deviance and Changes, Nueva York: Guilford Press.

Collins, M. E. (1991). Body figure perceptions and preferences among preadolescent children. International Journal of Eating Disorders, 10(2), 199-208. 
Contreras, O.R., Fernández, J., Garciìa, L. M., Palou, P., y Ponseti, J. (2010). El autoconcepto fiìsico y su relacioìn con la praìctica deportiva en estudiantes adolescentes. Revista de Psicologiìa del Deporte, 19(1), 23-29.

De Gracia, M., Marcó, M., y Trujano, P. (2007). Factores asociados a la conducta alimentaria en preadolescentes. Psicothema, 19(4), 646-653.

Del Pozo Cruz, J., Piedra, J., y Alfonso R. M. (2017). Influencia del género, edad, nivel de competición y disciplina en la imagen corporal de jóvenes gimnastas. Habilidad motriz: Revista de ciencias de la actividad física y del deporte, (48), 5-14.

Dowdell, T. (2013). Benefits of gymnastics participation for school age children. Education, 16, 1-17.

Fernández, L. G., y Azofeifa, E. G. (2007). Relación entre la satisfacción con la imagen corporal autoconcepto físico, índice de masa corporal y factores socioculturales en mujeres adolescentes costarricenses. PENSAR EN MOVIMIENTO: Revista de Ciencias del Ejercicio y la Salud, 5(1), 9-18.

Gómez, D. M., y Núñez, O. V. (2007). Insatisfacción corporal en adolescentes: relaciones con la actividad física e índice de masa corporal. Revista Internacional de Medicina y Ciencias de la Actividad Física y del Deporte/ International Journal of Medicine and Science of Physical Activity and Sport, 7(27), 253-264.

González-Montero de Espinosa, M., André, A. L., GarcíaPetuya, E., López-Ejeda, N., Mora, A. I. y Marrodán, M. D. (2010). Asociación entre actividad física y percepción de la imagen corporal en adolescentes madrileños. $\mathrm{Nu}$ trición clínica y dietética hospitalaria, 30(3), 4-12.

Janssen, I., y Le Blanc, A. G. (2010). Systematic review of the health benefits of physical activity and fitness in schoolaged children and youth. International journal of behavioral nutrition and physical activity, 7(1), 40.

Maganto C. y Cruz S. (2008). Test de siluetas para adolescentes. Madrid: TEA ediciones SA.

Marrodán, M.D., Montero-Roblas, V., Mesa, M. S., Pacheco, J. Gonzailez, M., y Carmenate, M. M. (2008). Realidad, percepcioìn y atractivo de la imagen corporal: condicionantes bioloìgicos y socioculturales. Zainak. Cuadernos de Antropología- Etnografía, 30(1), 15-28.

Miñano, M. J. C., García, E. F., y Galiano, M. I. R. (2006). Imagen corporal y práctica de actividad física en las chicas adolescentes: Incidencia de la modalidad deportiva. RICYDE. Revista Internacional de Ciencias del Deporte, 2(3), 1-19.

O'Dea, J.A., y Abraham, S. (2000). Improving the body image, eating attitudes, and behaviors of young male and female adolescents: A new educational approach that focuses on self esteem. International Journal of Eating Disorders, 28(1), 43-57.

Peláez, E.M., y Santana, M. (2018). Estima Corporal y factores antropométricos en adolescentes practicantes de acrobática. TRANCES. Transmisión del Conocimiento Educativo y de la Salud, (1), 511-526.

Quiles, Y., Quiles, M.J., Pamies, L., Botella, J. y Treasure, J. (2013). Peer and family influence in eating disorders: A meta-analysis. European Psychiatry, 28, 199-206.

Reguera, X., y Gutiérrez-Sánchez, A. (2015). Implementacioìn de un programa de Gimnasia Acrobaitica en Educacioìn Secundaria para la mejora del autoconcepto. Retos, (27), $114-117$

Stunkard, A., y Stellar, E. (1990). Eating and its disorderes. En Cash, T., Pruzinsky, T. (Eds): Body Images. Guilford Press: 3-20. Nueva York.

Taboada, Y., Vernetta, M., y Gutiérrez-Sánchez, A. (2017). Anthropometric Profile in Different Event Categories of Acrobatic Gymnastics. Journal of Human Kinetics, 57, 169-179.

Trejo Ortiz, P. M., Castro Veloz, D., Facio Solís, A., Mollinedo Montano, F. E., y Valdez Esparza, G. (2010). Insatisfacción con la imagen corporal asociada al Índice de Masa Corporal en adolescentes. Revista Cubana de Enfermería, 26(3), 150-160.

Tylka, T. L., y Wood-Barcalow, N. L. (2015). What is and what is not positive body image? Conceptual foundations and construct definition, Body Image, 14, 118-129.

Urrutia, S., Azpillaga, I., Luis de Cos, G., y Muñoz, D. (2010). Relación entre la percepción del estado de salud con la práctica físico-deportiva y la imagen corporal en adolescentes. Cuadernos de Psicología del Deporte, 10(Suple.), 51-56.

Valles, G., Hernández, E., Balos, R., Moncada-Jiménez, J. y Rentería, I. (2020) Distorsión de la imagen corporal y trastornos alimentarios en adolescentes gimnastas respecto a un grupo de control de adolescentes no gimnastas con un IMC similar. Retos: nuevas tendencias en educación física, deporte y recreación, 37(1), 297-302.

Vaquero-Cristóbal, R., Alacid, F., Muyor, J. M., y LópezMiñarro, P.A. (2013). Imagen corporal: revisión bibliográfica. Nutrición hospitalaria, 28(1), 27-35.

Vernetta, M., Fernández, E., López-Bedoya, J., GómezLandero, A., y Oña, A. (2011). Estudio relacional entre el perfil morfológico y estima corporal en la selección andaluza de gimnasia rítmica. Motricidad. European Journal of Human Movement, 26, 77-92.

Vernetta, M., López, J., y Gutiérrez, A. (2008). La creatividad de la gimnasia acrobaitica. En A. Martínez y P. Díaz (Coord.), Creatividad y deporte. Consideraciones teóricas e investigaciones breves (pp. 133-156). Sevilla: Wanceulen.

Vernetta, M., López Bedoya, J., y Panadero, F. (2007). El Acrosport en la escuela (3a ed.). Barcelona: Inde.

Vernetta, M., Montosa, I., y Peláez, E. (2018). Estima corporal en gimnastas adolescentes de dos disciplinas coreograìficas: gimnasia riitmica y gimnasia acrobaitica. Psychology, Society, \& Education, 10(3), 301-314.

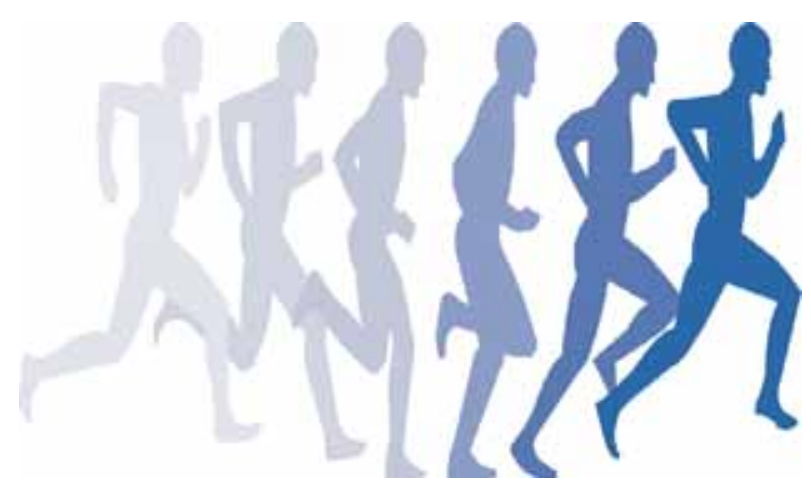

